\title{
The Development of Guillain-Barre Syndrome (Gbs) in Association with Confirmed Lyme Disease. A Potential Autoimmune Response in Gbs Secondary to Tick-Borne Diseases?
}

\author{
Seemal F Awan and Frederick T Murphy \\ Penn State College of Medicine, Pennsylvania ,USA
}

*Corresponding author: Frederick T Murphy, Penn State College of Medicine, Pennsylvania ,USA, Tel: +1-717-531-8521; E-mail: ftmurphy8@gmail.com Received date: March 03, 2015; Accepted date: May 08, 2015; Published date: May 15, 2015

Copyright: (C) 2015 Awan SF, et al. This is an open-access article distributed under the terms of the Creative Commons Attribution License, which permits unrestricted use, distribution, and reproduction in any medium, provided the original author and source are credited.

\section{Abstract}

Vector borne diseases have been associated with a number of autoimmune diseases. In this case we report a 73 year old woman initially diagnosed with lyme disease who subsequently developed guillain-barre syndrome (GBS). Her relevant clinical presentation included protean symptoms of numbness and progressive weakness in her legs for three weeks prior to consultation by the neurology service. A lyme ELISA and confirmatory Western Blot testing were unequivocally positive. A clinical diagnosis of CNS lyme disease was made. Additional clinical evaluation included: electrophysiological testing, which demonstrated significant polyneuropathy consistent with demyelinating pathology. A lumbar puncture with cerebral spinal fluid analysis revealed a non-reactive VDRL, negative lyme DNA PCR, positive lyme IgG antibody and an elevated protein-elevated albumin with normal white count. Upon further clinical deliberation this constellation of signs and symptoms was determined to be more consistent with a diagnosis of GBS rather than CNS lyme disease. Initiation of medical therapy included IVIG (intravenous immunoglobulin) and parenteral ceftriaxone. This case may report an additional vector of disease in patients presenting with clinical signs and symptoms of GBS.
\end{abstract}

Keywords: Guillain-barre syndrome; Cytomegalovirus; Lyme disease; Neuroborreliosis

\section{Introduction}

Guillain-barre syndrome (GBS) is an acute immune-mediated polyneuropathy that usually presents with progressive ascending weakness $[1,2]$. It has been recently understood that an antecedent infection may lead to the development of GBS. Two recently reported infectious diseases; Campylobacter and Cytomegalovirus have been cited as causative agents in GBS [3]. It is now accepted that an infectious disease may predicate an immune response resulting in the development of GBS [3]. Our patient may represent documentation of another causative agent in the development of GBS. Tick borne diseases such as Borrelia burgdorferi, have been identified as a causative agent in lyme disease in humans. Lyme disease may have protean immune modulating manifestations involving multiple organ systems including the skin, joints, heart, and brain. Neuroborreliosis, a common presentation of CNS lyme disease is characterized by peripheral and cranial neuropathies, which may also be a presenting symptom of GBS [4]. This particular case, reports a possible association of $B$. burgdorgeri as an inciting agent for GBS.

\section{Case Report}

A 73 year old Caucasian woman with a clinical history of hypertension and chronic obstructive pulmonary disease (COPD) presented to the Emergency Department with three weeks of progressive numbness and weakness in her lower extremities bilaterally and numbness noted in her chest and abdomen. This is a patient who prior to this clinical presentation lives by herself, was independent, was able to perform activities of daily living (ADL) without assistance, and was able to engage in recreational activities.

One week prior to presentation in the $\mathrm{ED}$, the patient noticed significant trouble with walking independently and proceeded to see a Neurologist. EMG (electromyogram) studies performed at that time showed significant polyneuropathy, involving both sensory and motor fibers, of demyelinating pathology. As part of an initial diagnostic evaluation, two-tier testing (Figure 1) was performed with a lyme antibody screen, which was positive with a subsequent positive Western Blot testing demonstrating 10 positive IgG bands and 1 positive IgM band. The patient was unaware of any prior exposure to lyme disease. A Lumbar puncture was also performed and notably negative for VDRL and lyme DNA PCR. There were IgG lyme antibodies detected. The gram stain and culture did not grow any organisms. The CSF did show an elevated protein of $98 \mathrm{mg} / \mathrm{dl}$ and an elevated albumin. The white blood cell count of $4 \mathrm{WBCs} / \mu \mathrm{l}$ was within normal limits. Magnetic resonance imaging (MRI) scan of the C-spine (cervical spine) showed degenerative joint disease and mild to moderate disk herniation $\mathrm{C} 3 / \mathrm{C} 4, \mathrm{C} 5 / \mathrm{C} 6$ without compression or myelopathy. MRI of the brain showed mild atrophy consistent with small vessel disease and encephalomalacia of the left occipital lobe, (Figures 2 and 3). The patient was started on IV ceftriaxone $2 \mathrm{~g}$ once daily for a total of 28 days.

One week after initiation of parenteral ceftriaxone, the patient reported a significant amount of pain in her back, noticed numbness to the level of her lips, and felt as if she was slurring her words. At this time, the patient was unable to move and could not participate in ADLs independently. The patient came to the ED whereby a lumbar puncture was performed which showed albuminocytologic dissociation consistent with GBS. The patient was consequently admitted to the hospital for IVIG treatment. 
Citation: Awan SF, Murphy FT (2015) The Development of Guillain-Barre Syndrome (Gbs) in Association with Confirmed Lyme Disease. A Potential Autoimmune Response in Gbs Secondary to Tick-Borne Diseases?. Clin Microbiol 4: 199. doi:10.4172/2327-5073.1000199

Page 2 of 4

The patient's hospital course was notable for administration of IVIG $0.4 \mathrm{~g} / \mathrm{kg}$ per day beginning on hospital day 2 with continuation of IVIG for a total of 6 days. The patient also continued to receive IV (intra-venous) ceftriaxone.

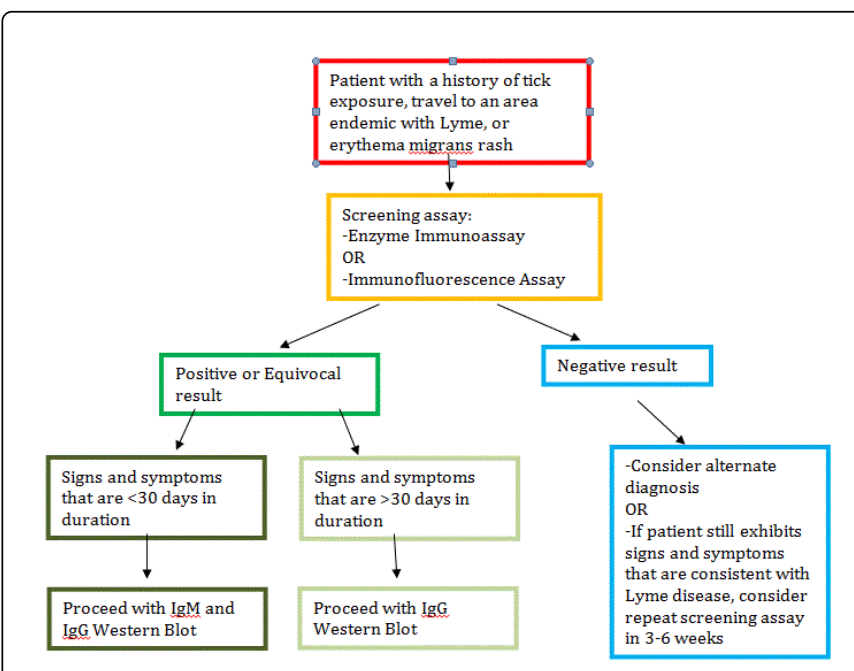

Figure 1: Two Tier Testing for lyme disease. It shows the steps that need to be taken in order to test for lyme disease. The first step is to perform enzyme immunoassay (EIA) or immunofluorescence assay (IFA). If the test that is performed is negative, an alternative diagnosis should be considered. However, a positive test would not confirm diagnosis and secondary testing would need to be perfomed. Based on the duration of symptoms, either IgM and IgG or only IgG western blot would need to be performed. A positive western blot would confirm the diagnosis.

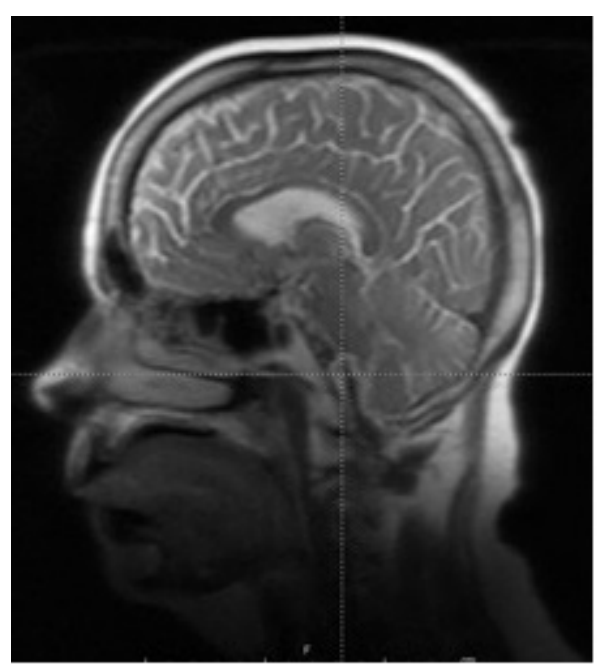

Figure 2: MRI Sagittal. It shows MRI of the patient one week prior to admission to the hospital. The MRI shows cerebral atrophy but no signs of an acute intracranial process.

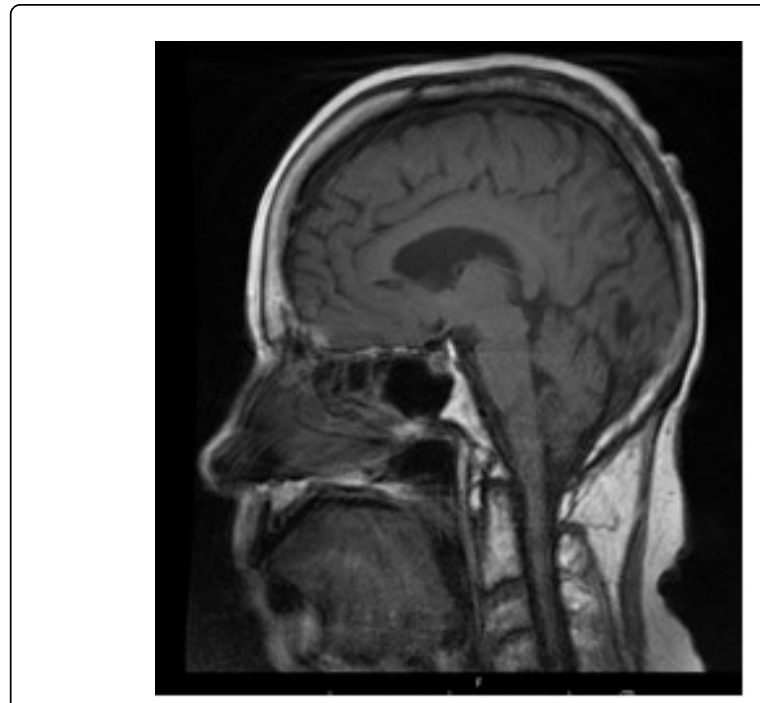

Figure 3: MRI Sagittal. It shows MRI of the patient one week prior to admission to the hospital. The MRI shows some encephalomalacia of the occipital lobe.

Due to the severity of pain in association with GBS, the patient was provided analgesic therapy. At the time of admission, examination of the musculoskeletal system revealed motor weakness with hip flexion graded $1 / 5$ bilaterally, and ankle dorsiflexion and planter flexion with a grade of $1 / 5$ bilaterally. There was ataxia with finger to nose bilaterally. Deep tendon reflexes were absent in lower extremities and 1+ in upper extremities. Light touch and temperature sensation were intact throughout the body.

During hospital day three, the patient did not notice the onset of any new symptoms but continued to need pain medications. On hospital day four, the patient did not have any change in her symptoms but was noted to have labile blood pressure. Vital signs revealed labile blood pressure readings ranging from $128 / 102 \mathrm{mmHg}$ to $178 / 74 \mathrm{mmHg}$. On hospital day five, day 4 of IVIG therapy, the patient reported a significant level of pain and had motor strength grading of $1 / 5$ in both legs. She was able to move her arms but had numbness up to her face. On hospital day six, day five of IVIG therapy, the patient had better pain management and was starting to regain function back in her lower extremities. She was able to dorsiflex and plantarflex her ankle and said the numbness in her face was down to below her neck. On hospital day seven, the final day of IVIG therapy, she was able to move her knees without assistance. Although residual numbness and tingling were still present, overall there was significant improvement in comparison to admission. On hospital day seven, persistent pain resulted in modification of medical therapy from Hydrocodone to Oxycodone. On hospital day eight, the patient had motor strength grading of $2-3 / 5$ in bilateral lower extremities. The reflexes now were detectable bilaterally in lower extremities. The patient's pain assessments continued to improve. The patient was discharged to an inpatient rehabilitation center for ongoing rehabilitation.

\section{Discussion}

Prior studies have demonstrated an incidence of Guillain-barre syndrome to be $1 / 100,000$, fortunately making it a rare and 
uncommon disease [5]. When first discovered in 1916, GBS was thought to be an isolated disease of unknown origin. Since that initial presentation, it has been established that GBS is a heterogeneous disease consisting of many different clinical presentations to include: acute inflammatory demyleniating polyradiculopathy (AIDP), the most common type in the western world, acute motor axonal neuropathy (AMAN), acute motor and sensory neuropathy (AMSAN), as well as other less common variants [1,5]. It is now widely accepted that a significant number of patients with GBS have experienced an antecedent infection. Campylobacter is the most common and widely known infection and about two-thirds of GBS patients report an infection with Campylobacter prior to the start of symptoms associated GBS [6]. This idea fueling Campylobacter as an inciting agent for GBS originates from the principle of molecular mimicry. Molecular mimicry is most simply described as when a foreign antigen is perceived as a self-antigen due to the presence of similar structural units $[6,8]$. It is thought that when humans are infected with Campylobacter, humans produce autoantibodies that target the Campylobacter ganglioside-like liposaccharrides and these liposaccharides are similar to gangliosides which are important in the function of Schwann cells, that myelinate peripheral nerves $[6,8]$. The time line associated with this sequence of the events seen in patients with GBS begin with the antecedent illness followed by production of antiglanglioside antibodies.

Similarly, this case study critically examines a possible association between Borrelia burgdorferi as a potential inciting agent for GBS. Presently there are few reported cases of an association between lyme disease and GBS. It is a widely accepted hypothesis that $B$. Burgdorferi is capable of producing an autoimmune response $[8,11]$. It is therefore plausible that the spirochete or components of the spirochete associated with lyme disease may also act as antigens or immune complexes, which facilitate production of antiglanglioside antibodies predicated in the development of GBS in susceptible individuals. If $B$. Burgdorferi may be involved in mounting an autoimmune response, then it is quite possible that it may also to be integral in reactive disease processes such as GBS. Further research in this area is warranted.

B. Burgdorferi is known best for the role it plays in causing the tickborne illness, lyme disease. Although B. Burgdorferi is not the only tick borne pathogen, it is definitely the most common pathogen known to result in lyme disease within the United States [11]. Lyme is a protean disease with systemic manifestations, which can target the skin, cardiovascular system, central nervous system and the musculoskeletal system. Lyme disease is further classified into distinct clinical stages $[11,12]$. The number of cases of documented lyme disease has been steadily increasing in the US. In the last ten years, there have been a total of 2,79,509 documented cases [11]. This number has increased by nearly $50 \%$ in the last decade [11]. As evidenced by these statistics, the occurrence of lyme disease is increasing. The prevalence of GBS may not be as well documented to the absence of a single diagnostic test. If the incidence of lyme disease is increasing and if the there is a potential association with this infectious disease and GBS then we may therefore surmise that a subset of patients with GBS could have had antecedent exposure to lyme disease. It would therefore be our recommendation that patients recently diagnosed with GBS also be tested for lyme disease.

A documented case report which may underscore this theory describes a woman who presented to the emergency department with an acute bilateral facial nerve palsy of unknown etiology [13]. Bilateral facial palsy is extremely rare with an incidence only 1 per 5 million patients. The most common infectious cause for this rare presentation is lyme disease $[13,14]$. It is therefore not by coincidence that lyme disease could be the causative agent for an immunologic disorder such as bilateral facial palsy $[11,14]$. This case along with other documented cases of chronic arthritis underscore that lyme disease may be strongly associated with diseases of the immune system and antecedent infection with lyme disease may be associated with GBS $[10,14]$.

\section{Conclusion}

Due to the rare nature of GBS, the difficulty in making an early diagnosis and challenges associated with appropriate medical management in the early course of disease all may portend a poor prognosis for the patient. The emphasis on appropriate diagnosis and early treatment are paramount. Therefore, searching for a potential antecedent infectious disease may not always be of primary clinical significance to the treating physician. The potential antecedent etiology may remain elusive if diagnostic studies are not considered early in the course of treatment. At the present time there is clear evidence of an association of an antecedent infectious disease associated with the development of GBS. This case study provides a unique clinical example whereby the presence of $B$. borgdorferi may have been an antecedent infectious disease associated with the development of GBS in this patient. The actual mechanism by which this process may occur is presently unclear. There is a documented immune response associated with tick borne pathogens. It is possible that these pathogens of components of the pathogen may serve as antigens or immune complexes in susceptible individuals resulting in the development of antiglanglioside antibodies. Further research may uncover the immunologic processes associated with GBS. This case report may establish a precedent for clinicians to consider antecedent lyme disease early in patients presenting to medical attention with signs and symptoms concerning for the development of Guillain-barre syndrome.

\section{References}

1. Vriesendorp FJ (2014) Clinical features and diagnosis of Guillain-Barré syndrome in adults. UpToDate.

2. Zhang J, Liu N, Zhang ZC, Zheng RZ, Li Q (2014) Sensory Guillain-Barre Syndrome: A Case Report. Exp Ther Med 8: 1713-1716.

3. Zabriskie, JB (2009) Essential Clinical Immunology. (1stedn.) Cambridge University Press, UK.

4. Schlossberg D (2008) Clinical Infectious Disease. (1stedn.) Cambridge University Press, UK.

5. Winer JB (2014) An update in guillain-barré syndrome. Autoimmune Dis 2014: 793024.

6. Nyati KK, Nyati R (2013) Role of Campylobacter jejuni Infection in the Pathogenesis of Guillain-Barré Syndrome: An Update. Biomed Res Int 2013.

7. van den Berg B, Walgaard C, Drenthen J, Fokke C, Jacobs BC, et al. (2014) Guillain-Barré syndrome: pathogenesis, diagnosis, treatment and prognosis. Nat Rev Neurol 10: 469-482.

8. Cusick MF, Libbey JE, Fujinami RS (2012) Molecular Mimicry as a Mechanism of Autoimmune Disease. Clin Rev Allergy Immunol 42: 102-111.

9. Sedzik J and Paolo R (2009) Molecules: Nucleation, Aggregation and Crystallization: Beyond Medical and Other Implications. World Scientific, Singapore.

10. Bolz DD, Weis JJ (2004) Molecular mimicry to Borrelia burgdorferi: pathway to autoimmunity? Autoimmunity $37: 387-392$. 
Citation: Awan SF, Murphy FT (2015) The Development of Guillain-Barre Syndrome (Gbs) in Association with Confirmed Lyme Disease. A Potential Autoimmune Response in Gbs Secondary to Tick-Borne Diseases?. Clin Microbiol 4: 199. doi:10.4172/2327-5073.1000199

Page 4 of 4

11. Beard CB (2014) Epidemiology of Lyme Disease. UpToDate.

12. Rashid T, Ebringer A (2012) Autoimmunity in Rheumatic Diseases Is Induced by Microbial Infections via Crossreactivity or Molecular Mimicry. Autoimmune Dis 2012: 539282.

13. Pothiawala S, Lateef F (2012) Bilateral Facial Nerve Palsy: A Diagnostic Dilemma. Case Reports in Emergency Medicine 2012.
14. Jain V, Deshmukh A, Gollomp S (2006) Bilateral Facial Paralysis Case Presentation and Discussion of Differential Diagnosis. J Gen Intern Med 21: C7-C10. 